\title{
A fixed-point approach to the stability of a functional equation on quadratic forms
}

\author{
Jae-Hyeong Bae and Won-Gil Park ${ }^{2^{*}}$
}

\author{
* Correspondence: \\ wgpark@mokwon.ac.kr \\ ${ }^{2}$ Department of Mathematics \\ Education, College of Education, \\ Mokwon University, Daejeon, 302- \\ 729, Korea \\ Full list of author information is \\ available at the end of the article
}

\section{Abstract}

Using the fixed-point method, we prove the generalized Hyers-Ulam stability of the functional equation

$$
f(x+y, z+w)+f(x-y, z-w)=2 f(x, z)+2 f(y, w) .
$$

The quadratic form $f: \mathbb{R} \times \mathbb{R} \rightarrow \mathbb{R}$ given by $f(x, y)=a x^{2}+b x y+c y^{2}$ is a solution of the above functional equation.

Keywords: alternative of fixed point, functional equation, quadratic form, stability

\section{Introduction}

In 1940, S. M. Ulam [1] gave a wide-ranging talk before the Mathematics Club of the University of Wisconsin in which he discussed a number of important unsolved problems. Among those was the question concerning the stability of group homomorphisms:

Let $G_{1}$ be a group and let $G_{2}$ be a metric group with the metric $d(\cdot, \cdot)$. Given $\varepsilon>0$, does there exist a $\delta>0$ such that if a function $h: G_{1} \rightarrow G_{2}$ satisfies the inequality $d(h$ $(x y), h(x) h(y))<\delta$ for all $x, y \in G_{1}$, then there is a homomorphism $H: G_{1} \rightarrow G_{2}$ with $d$ $(h(x), H(x))<\varepsilon$ for all $x \in G_{1}$ ?

The case of approximately additive mappings was solved by D. H. Hyers [2] under the assumption that $G_{1}$ and $G_{2}$ are Banach spaces. Thereafter, many authors investigated solutions or stability of various functional equations (see [3-11]).

Let $X$ be a set. A function $d: X \times X \rightarrow[0, \infty]$ is called a generalized metric on $X$ if $d$ satisfies

(1) $d(x, y)=0$ if and only if $x=y$;

(2) $d(x, y)=d(y, x)$ for all $x, y \in X$;

(3) $d(x, z) \leq d(x, y)+d(y, z)$ for all $x, y, z \in X$.

Note that the only substantial difference of the generalized metric from the metric is that the range of generalized metric includes the infinity.

Throughout this paper, let $X$ and $Y$ be two real vector spaces and let $\phi: X \times X \times X \times$ $X \rightarrow[0, \infty)$ be a function. For a mapping $f: X \times X \rightarrow Y$, consider the functional equation:

$$
f(x+y, z+w)+f(x-y, z-w)=2 f(x, z)+2 f(y, w) .
$$

(c) 2011 Bae and Park; licensee Springer. This is an Open Access article distributed under the terms of the Creative Commons Attribution License (http://creativecommons.org/licenses/by/2.0), which permits unrestricted use, distribution, and reproduction in any medium, provided the original work is properly cited. 
The quadratic form $f: \mathbb{R} \times \mathbb{R} \rightarrow \mathbb{R}$ given by $f(x, y):=a x^{2}+b x y+c y^{2}$ is a solution of the Equation 1.1.

The authors [12] acquired the general solution and proved the stability of the functional Equation 1.1 for the case that $X$ and $Y$ are real vector spaces as follows.

Theorem A. A mapping $f: X \times X \rightarrow Y$ satisfies the Equation 1.1 for all $x, y, z, w \in X$ if and only if there exist two symmetric bi-additive mappings $S, T: X \times X \rightarrow Y$ and a bi-additive mapping $B: X \times X \rightarrow Y$ such that

$$
f(x, y)=S(x, x)+B(x, y)+T(y, y)
$$

for all $x, y \in X$.

From now on, let $Y$ be a complete normed space.

Theorem B. Assume that $\phi$ satisfies the condition

$$
\tilde{\varphi}(x, y, z, w):=\sum_{j=0}^{\infty} \frac{1}{4^{j+1}} \varphi\left(2^{j} x, 2^{j} y, 2^{j} z, 2^{j} w\right)<\infty
$$

for all $x, y, z, w \in X$. Let $f: X \times X \rightarrow Y$ be a mapping such that

$$
\|f(x+y, z+w)+f(x-y, z-w)-2 f(x, z)-2 f(y, w)\| \leq \varphi(x, y, z, w)
$$

for all $x, y, z, w \in X$. Then, there exists a unique mapping $F: X \times X \rightarrow Y$ satisfying the Equation 1.1 such that

$$
\|f(x, y)-F(x, y)\| \leq \tilde{\varphi}(x, x, y, y)
$$

for all $x, y \in X$. The mapping $F$ is given by

$$
F(x, y):=\lim _{j \rightarrow \infty} \frac{1}{4^{j}} f\left(2^{j} x, 2^{j} y\right)
$$

for all $x, y \in X$.

In this paper, we prove the stability of the Equation 1.1 using the fixed-point method.

\section{Stability using the alternative of fixed point}

In this section, we investigate the stability of the functional Equation 1.1 using the alternative of fixed point. Before proceeding the proof, we will state the theorem, the alternative of fixed point.

Theorem 2.1. (The alternative of fixed point $[13,14])$. Suppose that we are given a complete generalized metric space $(\Omega, d)$ and a strictly contractive mapping $T: \Omega \rightarrow$ $\Omega$ with Lipschitz constant $L$. Then, for each given $x \in \Omega$, either

$$
d\left(T^{n} x, T^{n+1} x\right)=\infty \text { for all } n \geq 0,
$$

or

there exists a positive integer $n_{0}$ such that

- $d\left(T^{n} x, T^{n+1} x\right)<\infty$ for all $n \geq n_{0}$;

- the sequence $\left(T^{n} x\right)$ is convergent to a fixed point $y^{*}$ of $T$;

- $y^{*}$ is the unique fixed point of $T$ in the set $\Delta=\left\{y \in \Omega \mid d\left(T^{n_{0}} x, y\right)<\infty\right\}$;

- $d\left(y, y^{*}\right) \leq \frac{1}{1-L} d(y, T y)$ for all $y \in \Delta$. 
Lemma 2.2. Let $\psi: X \times X \rightarrow[0, \infty)$ be a function given by

$$
\psi(x, y):=\varphi\left(\frac{x}{2}, \frac{x}{2}, \frac{y}{2}, \frac{y}{2}\right)
$$

for all $x, y \in X$. Consider the set $\Omega:=\{g \mid g: X \times X \rightarrow Y, g(0,0)=0\}$ and the generalized metric $d$ on $\Omega$ given by

$$
d(g, h)=d_{\psi}(g, h):=\operatorname{infS}_{\psi}(g, h),
$$

where $S_{\psi}(g, h):=\{K \in[0, \infty] \mid\|g(x, y)-h(x, y)\| \leq K \psi(x, y)$ for all $x, y \in X\}$ for all $g, h \in \Omega$. Then, $(\Omega, d)$ is complete.

Proof. Let $\left\{g_{n}\right\}$ be a Cauchy sequence in $(\Omega, d)$. Then, given $\varepsilon>0$, there exists $N$ such that $d\left(g_{n}, g_{k}\right)<\varepsilon$ if $n, k \geq N$. Let $n, k \geq N$. Since $d\left(g_{n}, g_{k}\right)=\inf S_{\psi}\left(g_{n}, g_{k}\right)<\varepsilon$, there exists $K \in[0, \varepsilon)$ such that

$$
\left\|g_{n}(x, y)-g_{k}(x, y)\right\| \leq K \psi(x, y) \leq \varepsilon \psi(x, y)
$$

for all $x, y \in X$. So, for each $x, y \in X,\left\{g_{n}(x, y)\right\}$ is a Cauchy sequence in $Y$. Since $Y$ is complete, for each $x, y \in X$, there exists $g(x, y) \in Y$ such that $g_{n}(x, y) \rightarrow g(x, y)$ as $n \rightarrow$ $\infty$ and $g(0,0)=0$. Thus, we have $g \in \Omega$. By (2.1), we obtain that

$$
\begin{aligned}
n \geq N & \Rightarrow\left\|g_{n}(x, y)-g(x, y)\right\| \leq \varepsilon \psi(x, y) \text { for all } x, y \in X \\
& \Rightarrow \varepsilon \in S_{\psi}\left(g_{n}, g\right) \\
& \Rightarrow d\left(g_{n}, g\right)=\operatorname{infS}_{\psi}\left(g_{n}, g\right) \leq \varepsilon .
\end{aligned}
$$

Hence, $g_{n} \rightarrow g \in \Omega$ as $n \rightarrow \infty$.

By using an idea of Cădariu and Radu (see [15]), we will prove the Hyers-Ulam stability of the functional equation related to quadratic forms.

Theorem 2.3. Assume that $\phi$ satisfies the condition

$$
\lim _{n \rightarrow \infty} \frac{1}{4^{n}} \varphi\left(2^{n} x, 2^{n} y, 2^{n} z, 2^{n} w\right)=0
$$

for all $x, y, z, w \in X$. Suppose that a mapping $f: X \times X \rightarrow Y$ satisfies the functional inequality

$$
\|f(x+y, z+w)+f(x-y, z-w)-2 f(x, z)-2 f(y, w)\| \leq \varphi(x, y, z, w)
$$

for all $x, y, z, w \in X$ and $f(0,0)=0$. If there exists $L<1$ such that the function $\psi$ given in Lemma 2.2 has the property

$$
\psi(x, y) \leq 4 L \psi\left(\frac{x}{2}, \frac{y}{2}\right)
$$

for all $x, y \in X$, then there exists a unique mapping $F: X \times X \rightarrow Y$ satisfying (1.1) such that the inequality

$$
\|f(x, y)-F(x, y)\| \leq \frac{L}{1-L} \psi(x, y)
$$

holds for all $x, y \in X$.

Proof. Consider the complete generalized metric space $(\Omega, d)$ given in Lemma 2.2. Now we define a mapping $T: \Omega \rightarrow \Omega$ by 


$$
\operatorname{Tg}(x, y):=\frac{1}{4} g(2 x, 2 y)
$$

for all $g \in \Omega$ and all $x, y \in X$. Observe that, for all $g, h \in \Omega$,

$$
\begin{aligned}
& K^{\prime} \in S_{\psi}(g, h) \text { and } K^{\prime}<K \\
& \quad \Rightarrow\|g(x, y)-h(x, y)\| \leq K^{\prime} \psi(x, y) \leq K \psi(x, y) \text { for all } x, y \in X \\
& \quad \Rightarrow K \in S_{\psi}(g, h) .
\end{aligned}
$$

Let $g, h \in \Omega$ and $\varepsilon \in(0, \infty]$. Then, there is a $K^{\prime} \in S_{\psi}(g, h)$ such that $K^{\prime}<d(g, h)+\varepsilon$. By the above observation, we gain $d(g, h)+\varepsilon \in S_{\psi}(g, h)$. So we get $\|g(x, y)-h(x, y)\| \leq$ $(d(g, h)+\varepsilon) \psi(x, y)$ for all $x, y \in X$. Thus, we have

$$
\left\|\frac{1}{4} g(2 x, 2 y)-\frac{1}{4} h(2 x, 2 y)\right\| \leq \frac{1}{4}(d(g, h)+\varepsilon) \psi(2 x, 2 y)
$$

for all $x, y \in X$. By (2.3), we obtain that

$$
\left\|\frac{1}{4} g(2 x, 2 y)-\frac{1}{4} h(2 x, 2 y)\right\| \leq L(d(g, h)+\varepsilon) \psi(x, y)
$$

for all $x, y \in X$. Hence, $d(T g, T h) \leq L(d(g, h)+\varepsilon)$. Now we obtain that

$$
d(T g, T h) \leq L(d(g, h)+\varepsilon)
$$

for all $\varepsilon \in(0, \infty]$. Taking the limit as $\varepsilon \rightarrow 0^{+}$in the above inequality, we get

$$
d(T g, T h) \leq L d(g, h)
$$

for all $g, h \in \Omega$, that is, $T$ is a strictly contractive mapping of $\Omega$ with Lipschitz constant $L$.

Putting $y=x$ and $w=z$ in (2.2), by (2.3), we have the inequality

$$
\left\|f(x, z)-\frac{1}{4} f(2 x, 2 z)\right\| \leq \frac{1}{4} \varphi(x, x, z, z)=\frac{1}{4} \psi(2 x, 2 z) \leq L \psi(x, z)
$$

for all $x, z \in X$. Thus, we obtain that

$$
d(f, T f) \leq L<\infty .
$$

Applying the alternative of fixed point, we see that there exists a fixed point $F$ of $T$ in $\Omega$ such that

$$
F(x, y)=\lim _{n \rightarrow \infty} \frac{1}{4^{n}} f\left(2^{n} x, 2^{n} y\right)
$$

for all $x, y \in X$. Replacing $x, y, z, w$ by $2^{n} x, 2^{n} y, 2^{n} z, 2^{n} w$ in (2.2), respectively, and dividing by $4^{n}$, we have

$$
\begin{aligned}
\| & F(x+y, z+w)+F(x-y, z-w)-2 F(x, z)-2 F(y, w) \| \\
= & \lim _{n \rightarrow \infty} \frac{1}{4^{n}} \| f\left(2^{n}(x+y), 2^{n}(z+w)\right)+f\left(2^{n}(x-y), 2^{n}(z-w)\right) \\
& \quad-2 f\left(2^{n} x, 2^{n} z\right)-2 f\left(2^{n} y, 2^{n} w\right) \| \\
\leq & \lim _{n \rightarrow \infty} \frac{1}{4^{n}} \varphi\left(2^{n} x, 2^{n} y, 2^{n} z, 2^{n} w\right)=0
\end{aligned}
$$


for all $x, y, z, w \in X$. Thus, the mapping $F$ satisfies the Equation 1.1. By (2.3) and (2.5), we obtain that

$$
\begin{aligned}
\left\|T^{n} f(x, y)-T^{n+1} f(x, y)\right\| & =\frac{1}{4^{n}}\left\|f\left(2^{n} x, 2^{n} y\right)-\frac{1}{4} f\left(2^{n+1} x, 2^{n+1} y\right)\right\| \\
& \leq \frac{L}{4^{n}} \psi\left(2^{n} x, 2^{n} y\right) \leq \cdots \leq \frac{L}{4^{n}}(4 L)^{n} \psi(x, y) \\
& =L^{n+1} \psi(x, y)
\end{aligned}
$$

for all $x, y \in X$ and all $n \in \mathbb{N}$, that is, $d\left(T^{n} f, T^{n+1} f\right) \leq L^{n+1}<\infty$ for all $n \in \mathbb{N}$. By the fixed-point alternative, there exists a natural number $n_{0}$ such that the mapping $F$ is the unique fixed point of $T$ in the set $\Delta=\left\{g \in \Omega \mid d\left(T^{n_{0}} f, g\right)<\infty\right\}$. So we have $d\left(T^{n_{0}} f, F\right)<\infty$. Since

$$
d\left(f, T^{n_{0}} f\right) \leq d(f, T f)+d\left(T f, T^{2} f\right)+\cdots+d\left(T^{n_{0}-1} f, T^{n_{0}} f\right)<\infty,
$$

we get $f \in \Delta$. Thus, we have $d(f, F) \leq d\left(f, T^{m_{0}} f\right)+d\left(T^{m_{0}} f, F\right)<\infty$. Hence, we obtain

$$
\|f(x, y)-F(x, y)\| \leq K \psi(x, y)
$$

for all $x \in X$ and some $K \in[0, \infty)$. Again using the fixed-point alternative, we have

$$
d(f, F) \leq \frac{1}{1-L} d(f, T f) .
$$

By (2.6), we may conclude that

$$
d(f, F) \leq \frac{L}{1-L},
$$

which implies the inequality (2.4).

Lemma 2.4. Let $\psi: X \times X \rightarrow[0, \infty)$ be a function given by

$$
\psi(x, y):=\varphi(2 x, 2 x, 2 y, 2 y)
$$

for all $x, y \in X$. Consider the set $\Omega:=\{g \mid g: X \times X \rightarrow Y, g(0,0)=0\}$ and the generalized metric $d$ on $\Omega$ given by

$$
d(g, h)=d_{\psi}(g, h):=\operatorname{infS}_{\psi}(g, h),
$$

where $S_{\psi}(g, h):=K \in[0, \infty] \mid\|g(x, y)-h(x, y)\| \leq K \psi(x, y)$ for all $\left.x, y \in X\right\}$ for all $g$, $h \in \Omega$. Then, $(\Omega, d)$ is complete.

Proof. The proof is similar to the proof of Lemma 2.2.

Theorem 2.5. Assume that $\phi$ satisfies the condition

$$
\lim _{n \rightarrow \infty} 4^{n} \varphi\left(\frac{x}{2^{n}}, \frac{y}{2^{n}}, \frac{z}{2^{n}}, \frac{w}{2^{n}}\right)=0
$$

for all $x, y, z, w \in X$. Suppose that a mapping $f: X \times X \rightarrow Y$ satisfies the functional inequality (2.2) for all $x, y, z, w \in X$ and $f(0,0)=0$. If there exists $L<1$ such that the function $\psi$ given in Lemma 2.4 has the property

$$
\psi(x, y) \leq \frac{L}{4} \psi(2 x, 2 y)
$$


for all $x, y \in X$, then there exists a unique mapping $F: X \times X \rightarrow Y$ satisfying (1.1) such that the inequality

$$
\|f(x, y)-F(x, y)\| \leq \frac{L^{2}}{16(1-L)} \psi(x, y)
$$

holds for all $x, y \in X$.

Proof. Consider the complete generalized metric space $(\Omega, d)$ given in Lemma 2.4. Now we define a mapping $T: \Omega \rightarrow \Omega$ by

$$
\operatorname{Tg}(x, y):=4 g\left(\frac{x}{2}, \frac{y}{2}\right)
$$

for all $g \in \Omega$ and all $x, y \in X$. By the same argument of the proof of Theorem 2.3, $T$ is a strictly contractive mapping of $\Omega$ with Lipschitz constant $L$.

Replacing $x, y, z, w$ by $\frac{x}{2}, \frac{x}{2}, \frac{z}{2}, \frac{z}{2}$ in (2.2), respectively, and using (2.7), we have the inequality

$$
\left\|f(x, z)-4 f\left(\frac{x}{2}, \frac{z}{2}\right)\right\| \leq \varphi\left(\frac{x}{2}, \frac{x}{2}, \frac{z}{2}, \frac{z}{2}\right)=\psi\left(\frac{x}{4}, \frac{z}{4}\right) \leq \frac{L}{4} \psi\left(\frac{x}{2}, \frac{z}{2}\right) \leq \frac{L^{2}}{16} \psi(x, y)
$$

for all $x, z \in X$. Thus, we obtain that

$$
d(f, T f) \leq \frac{L^{2}}{16}<\infty
$$

Applying the alternative of fixed point, we see that there exists a fixed point $F$ of $T$ in $\Omega$ such that

$$
F(x, y)=\lim _{n \rightarrow \infty} 4^{n} f\left(\frac{x}{2^{n}}, \frac{y}{2^{n}}\right)
$$

for all $x, y \in X$. Replacing $x, y, z, w$ by $\frac{x}{2^{n}}, \frac{y}{2^{n}}, \frac{z}{2^{n}}, \frac{w}{2^{n}}$ in (2.2), respectively, and multiplying by $4^{n}$, we have

$$
\begin{aligned}
& \|F(x+y, z+w)+F(x-y, z-w)-2 F(x, z)-2 F(y, w)\| \\
& \quad=\lim _{n \rightarrow \infty} 4^{n}\left\|f\left(\frac{x+y}{2^{n}}, \frac{z+w}{2^{n}}\right)+f\left(\frac{x-y}{2^{n}}, \frac{z-w}{2^{n}}\right)-2 f\left(\frac{x}{2^{n}}, \frac{z}{2^{n}}\right)-2 f\left(\frac{y}{2^{n}}, \frac{w}{2^{n}}\right)\right\| \\
& \quad \leq \lim _{n \rightarrow \infty} 4^{n} \varphi\left(\frac{x}{2^{n}}, \frac{y}{2^{n}}, \frac{z}{2^{n}}, \frac{w}{2^{n}}\right)=0
\end{aligned}
$$

for all $x, y, z, w \in X$. Thus, the mapping $F$ satisfies the Equation 1.1. By (2.7) and (2.9), we obtain that

$$
\begin{aligned}
\left\|T^{n} f(x, y)-T^{n+1} f(x, y)\right\| & =4^{n}\left\|f\left(\frac{x}{2^{n}}, \frac{y}{2^{n}}\right)-4 f\left(\frac{x}{2^{n+1}}, \frac{y}{2^{n+1}}\right)\right\| \\
& \leq 4^{n-2} L^{2} \psi\left(\frac{x}{2^{n}}, \frac{y}{2^{n}}\right) \leq 4^{n-3} L^{3} \psi\left(\frac{x}{2^{n-1}}, \frac{y}{2^{n-1}}\right) \\
& \leq \cdots \leq \frac{L^{n+2}}{16} \psi(x, y)
\end{aligned}
$$

for all $x, y \in X$ and all $n \in \mathbb{N}$, that is, $d\left(T^{n} f, T^{n+1} f\right) \leq \frac{L^{n+2}}{16}<\infty$ for all $n \in \mathbb{N}$. By the same reasoning of the proof of Theorem 2.3, we have 


$$
d(f, F) \leq \frac{1}{1-L} d(f, T f)
$$

By (2.10), we may conclude that

$$
d(f, F) \leq \frac{L^{2}}{16(1-L)}
$$

which implies the inequality (2.8).

\section{Acknowledgements}

The authors would like to thank the referee for a number of valuable suggestions regarding a previous version of this paper.

\section{Author details}

${ }^{1}$ Graduate School of Education, Kyung Hee University, Yongin, 446-701, Korea ${ }^{2}$ Department of Mathematics Education, College of Education, Mokwon University, Daejeon, 302-729, Korea

\section{Authors' contributions}

Both authors contributed equally to this work. The authors read and approved the final manuscript.

\section{Competing interests}

The authors declare that they have no competing interests.

Received: 15 April 2011 Accepted: 10 October 2011 Published: 10 October 2011

\section{References}

1. Ulam, SM: A Collection of Mathematical Problems. pp. 63. Interscience Publishers, New York (1968)

2. Hyers, DH: On the stability of the linear functional equation. Proc Natl Acad Sci USA. 27, 222-224 (1941). doi:10.1073/ pnas.27.4.222

3. Bae, J-H, Park, W-G: On stability of a functional equation with $n$ variables. Nonlinear Anal TMA. 64, 856-868 (2006). doi:10.1016/j.na.2005.06.028

4. Bae, J-H, Park, W-G: On a cubic equation and a Jensen-quadratic equation. Abstr Appl Anal 2007 (2007). Article ID 45179

5. Găvruta, P: A generalization of the Hyers-Ulam-Rassias stability of approximately additive mappings. J Math Anal Appl. 184, 431-436 (1994). doi:10.1006//maa.1994.1211

6. Jung, S-M: Hyers-Ulam-Rassias Stability of Functional Equations in Nonlinear Analysis. Springer, New York (2011)

7. Jung, S-M, Kim, T-S: A fixed point approach to the stability of the cubic functional equation. Bol Soc Mat Mexicana 12(1), 51-57 (2006). (3)

8. Jung, S-M, Kim, T-S, Lee, K-S: A fixed point approach to the stability of quadratic functional equation. Bull Korean Math Soc. 43, 531-541 (2006)

9. Jung, S-M, Lee, Z-H: A fixed point approach to the stability of quadratic functional equation with involution. Fixed Point Theory Appl 2008, 11 (2008). Article ID 732086

10. Park, W-G, Bae, J-H: A multidimensional functional equation having quadratic forms as solutions. J Inequal Appl 2007 (2007). Article ID 24716

11. Rassias, TM: On the stability of linear mappings in Banach spaces. Proc Amer Math Soc. 72, 297-300 (1978). doi:10.1090/ S0002-9939-1978-0507327-1

12. Park, W-G, Bae, J-H: A functional equation originating from quadratic forms. J Math Anal Appl. 326, 1142-1148 (2007). doi:10.1016/j.jmaa.2006.03.023

13. Margolis, B, Dias, JB: A fixed point theorem of the alternative for contractions on a generalized complete metric space. Bull Amer Math Soc. 74, 305-309 (1968). doi:10.1090/50002-9904-1968-11933-0

14. Rus, IA: Principles and Applications of Fixed Point Theory. (1979) in Romanian

15. Cădariu, L, Radu, V: Fixed points and the stability of Jensen's functional equation. J Inequal Pure Appl Math 4 (2003). Article 4

doi:10.1186/1029-242X-2011-82

Cite this article as: Bae and Park: A fixed-point approach to the stability of a functional equation on quadratic

forms. Journal of Inequalities and Applications 2011 2011:82. 